\title{
Testing Earnings Management
}

\author{
Dennis Fok \\ Philip Hans Franses \\ Econometric Institute \\ Erasmus School of Economics
}

Econometric Institute Report 2009-31

\begin{abstract}
Earnings management to avoid earnings decreases and losses implies that the time series properties of the last quarter in the fiscal year differ from those of the other three quarters. We propose a simple parametric methodology to diagnose such differences. Application to a random sample of 390 firms in the Compustat database gives strong evidence of earnings management.
\end{abstract}

Key words: Earnings management; periodic time series models

JEL classification: M41; M43

This version: October 21, 2009

\section{Authors' notes}

We thank Rogier Potter van Loon for excellent research assistance. Address for correspondence is Econometric Institute, Erasmus School of Economics, PO Box 1738, 3000 DR Rotterdam, The Netherlands, dfok@ese.eur.nl, franses@ese.eur.nl 


\section{Introduction}

The use of earnings management to avoid earnings decreases and/or losses is never directly observed, and any evidence for this phenomenon should be deduced from observable earnings data. In the relevant literature, there are two dominant approaches to deduce earnings management. The first looks at discontinuities of the earnings distribution around zero. This approach adopts non-parametric techniques to analyze histograms of earnings data, see the important studies of Hayn (1995), Burgstahler and Dichev (1997), DeGeorge et al. (1999) and the recent study in Jacob and Jorgenson (2007). The second approach specifically focuses at the data on the final quarter of the fiscal year to see if this quarter shows other properties than the other quarters, see Collins et al. (1984) and more recently Das et al. (2007) and Hayn et al. (2007). In the present paper we aim to contribute to this second stream of literature as we have a tool that is useful to elicit differing properties of the final quarter data. .

In this paper we demonstrate that earnings management implies specific time series properties of the fiscal year's final quarter data (in our empirical study we zoom in on fourth quarter of the year). We show that these properties can be captured in a periodic time series model. Such a model allows some parameters to vary with the seasons, see Franses (1996) and Franses and Paap (2004) for introductory textbooks, and we will show that earnings management predicts a particular pattern in these seasonally varying parameters. To allow for the apparent heterogeneity across firms, we propose a two-level model, with as first level the periodic time series model, and with as second level the distributions of the parameters. We analyze this two-level model using Bayesian routines, more specifically, using Markov chain Monte Carlo techniques.

The outline of our paper is as follows. In Section 2 we outline the main idea of our study, that is, the link between earnings management and a periodic time series model. In Section 3 we propose a simple methodology that can be used to diagnose if final quarter earnings data have properties that differ from other quarters' data. In Section 4 we apply our methodology to a random sample of 390 firms from the Compustat database, all of which have quarter 4 as the last quarter of the fiscal year, and for which we have data for 1980Q1 to 2001Q4. Using four variants of the periodic model, we find overwhelming evidence of earnings management. We 
formally compare the models using Bayes factors. Section 5 concludes this paper with various topics for further research.

\section{The main idea}

Earnings management implies time series properties of the final quarter in a fiscal year that differ from those of the other three quarters. In a seminal paper, Collins et al. (1984) already diagnosed that fiscal year's final quarter data are more difficult to forecast than other quarters' data. This notion naturally translates to the phenomenon that final quarter data perhaps obey another time series model than the other three quarters. This feature can be captured by a so-called periodic time series model, and in this section we demonstrate this link.

\subsection{Modelling quarterly earnings data}

To save notation, we assume that the fiscal year's last quarter is the fourth quarter. When this is not the case, one can simply rearrange the data by relabeling the quarters. We use a simple time series model, but we stress that for any other time series model the same qualitative results will be obtained.

Suppose that, in the absence of earnings management, the model for quarterly earnings data for a firm $i=1, \ldots, K$ is an autoregression of order 1 [AR(1)], which reads as

$$
y_{i, t}=\mu_{i, s_{t}}+\rho_{i} y_{i, t-1}+\varepsilon_{i, t} .
$$

The variable $y_{i, t}$ denotes the quarterly earnings data, where $t=1,2, \ldots, n=4 N$, with $N$ is the number of years of available data. The indicator $s_{t}$ gives the quarter corresponding to observation $t$. The parameter $\mu_{i, s}$ denotes an intercept that can vary with the season $s$, where $\mathrm{s}=1,2,3$ or 4 . The parameter $\rho_{i}$ gives the autoregressive parameter for firm $i$. Finally, the error term $\varepsilon_{i, t}$ is distributed as normal with mean 0 and variance $\sigma_{i}^{2}$. 
The autoregression in (1) is written in terms of quarterly data, but it can also be written in annual format. This can be achieved by denoting $Y_{i, s, T}$ as the observation in quarter $s$ in year $T$. Adopting this notation it is not difficult to see that (1) can be written as

$$
\begin{aligned}
& Y_{i, 1, T}=\mu_{i, 1}+\rho_{i} Y_{i, 4, T-1}+\varepsilon_{i, 1, T} \\
& Y_{i, 2, T}=\mu_{i, 2}+\rho_{i} Y_{i, 1, T}+\varepsilon_{i, 2, T} \\
& Y_{i, 3, T}=\mu_{i, 3}+\rho_{i} Y_{i, 2, T}+\varepsilon_{i, 3, T} \\
& Y_{i, 4, T}=\mu_{i, 4}+\rho_{i} Y_{i, 3, T}+\varepsilon_{i, 4, T} .
\end{aligned}
$$

The four equations above each correspond to a particular quarter of the year. The observation for the first quarter in year $T$ is obviously related to the fourth quarter in year $T-1$ (first line in (2)). The four error terms $\varepsilon_{i, s, T}$ are distributed as normal with mean 0 and common variance $\sigma_{i}^{2}$. Further note that the four equations share a common autoregressive parameter.

In case of earnings management, the data in the fourth quarter (again assuming that this is the relevant quarter) will have properties that differ from those of the other three quarters. First of all, the unobserved error term $\varepsilon_{i, 4, T}$ will not fully capture the unexplained variation as earnings management will add an extra (unobserved to the analyst) term, say, $e_{i, 4, T}$. This feature explains the findings in Collins et al. (1984), as now indeed the forecast error variance for the fourth quarter is larger that that in other quarters, making it harder to predict. Second, when the fourth quarter data get an extra unobserved input, the correlation between a year's first quarter and the previous year's fourth quarter must change. In fact, it shall not be $\rho_{i}$ anymore but $\rho_{i, 1}$.

In sum, in case of earnings management, the four-equation model in (2) thus becomes

$$
\begin{aligned}
& Y_{i, 1, T}=\mu_{i, 1}+\rho_{i, 1} Y_{i, 4, T-1}+\varepsilon_{i, 1, T} \\
& Y_{i, 2, T}=\mu_{i, 2}+\rho_{i} Y_{i, 1, T}+\varepsilon_{i, 2, T} \\
& Y_{i, 3, T}=\mu_{i, 3}+\rho_{i} Y_{i, 2, T}+\varepsilon_{i, 3, T} \\
& Y_{i, 4, T}=\mu_{i, 4}+\rho_{i} Y_{i, 3, T}+\varepsilon_{i, 4, T}+e_{4, i, T} .
\end{aligned}
$$


This model is a restricted version of a so-called periodic autoregression of order 1, where all autoregressive parameters and all four variances are allowed to be season specific. This type of model is ich in its general expression is given by

$$
\begin{array}{ll}
Y_{i, 1, T}=\mu_{i, 1}+\rho_{i, 1} Y_{i, 4, T-1}+\varepsilon_{i, 1, T} & \varepsilon_{i, 1, T} \sim N\left(0, \sigma_{i, 1}^{2}\right) \\
Y_{i, 2, T}=\mu_{i, 2}+\rho_{i, 2} Y_{i, 1, T}+\varepsilon_{i, 2, T} & \varepsilon_{i, 2, T} \sim N\left(0, \sigma_{i, 2}^{2}\right) \\
Y_{i, 3, T}=\mu_{i, 3}+\rho_{i, 3} Y_{i, 2, T}+\varepsilon_{i, 3, T} & \varepsilon_{i, 3, T} \sim N\left(0, \sigma_{i, 3}^{2}\right) \\
Y_{i, 4, T}=\mu_{i, 4}+\rho_{i, 4} Y_{i, 3, T}+\varepsilon_{i, 4, T} & \varepsilon_{i, 4, T} \sim N\left(0, \sigma_{i, 4}^{2}\right) .
\end{array}
$$

Franses (1996) and Franses and Paap (2004) are introductory textbooks on this type of model. Representation of, inference in, and forecasting from such periodic models are similar to of the corresponding techniques for vector autoregressive models.

\subsection{Testable hypotheses}

In our illustration below, we will impose the restrictions $\rho_{i, 2}=\rho_{i, 3}=\rho_{i, 4}=\rho_{i}$ and $\sigma_{i, 2}^{2}=\sigma_{i, 3}^{2}=\sigma_{i, 1}^{2}=\sigma_{i}^{2}$ in (4). To test earnings management we simply examine the empirical validity of the following two hypotheses, that is,

Hypothesis 1: $\rho_{i, 1}=\rho_{i}$

Hypothesis 2: $\sigma_{i, 4}^{2}=\sigma_{i}^{2}$

If both hypotheses are true there is no evidence for earnings management. Note that the alternative hypotheses are $\rho_{\mathrm{i}, 1}<\rho_{\mathrm{i}}$ for hypothesis 1 and $\sigma_{i, 4}^{2}>\sigma_{i}^{2}$ for hypothesis 2 .

\section{Methodology}

In this section we first discuss the representation of the model and we describe how the parameters can be estimated. Next, we discuss hypothesis testing. 


\subsection{Representation and inference}

We consider the periodic time series model

$$
\begin{array}{ll}
Y_{i, 1, T}=\mu_{i, 1}+\rho_{i, 1} Y_{i, 4, T-1}+\varepsilon_{i, 1, T} & \varepsilon_{i, 1, T} \sim N\left(0, \sigma_{i}^{2}\right) \\
Y_{i, 2, T}=\mu_{i, 2}+\rho_{i} Y_{i, 1, T}+\varepsilon_{i, 2, T} & \varepsilon_{i, 2, T} \sim N\left(0, \sigma_{i}^{2}\right) \\
Y_{i, 3, T}=\mu_{i, 3}+\rho_{i} Y_{i, 2, T}+\varepsilon_{i, 3, T} & \varepsilon_{i, 3, T} \sim N\left(0, \sigma_{i}^{2}\right) \\
Y_{i, 4, T}=\mu_{i, 4}+\rho_{i} Y_{i, 3, T}+\varepsilon_{i, 4, T} & \varepsilon_{i, 4, T} \sim N\left(0, \sigma_{i 4}^{2}\right)
\end{array}
$$

For the vector of firm-specific parameters $\theta_{i}=\left(\mu_{i 1}, \mu_{i 2}, \mu_{i 3}, \mu_{i 4}, \rho_{i 1}, \rho_{i}\right)$ ' we specify

$$
\theta_{i} \sim N(\bar{\theta}, \Sigma)
$$

that is, the individual specific parameters have a joint multivariate normal distribution.

Inference is done via Markov Chain Monte Carlo techniques, especially we use Gibbs sampling (Geman and Geman 1984). Details of the sampling procedures are presented in the appendix. For model comparison and hypothesis testing it is important to set proper priors on $\bar{\theta}, \sigma_{i}^{2}, \sigma_{\mathrm{i} 4}^{2}$ and $\Sigma$. We set the priors to be proper but relatively uninformative.

We consider three restricted forms of this model. First of all we consider the restriction $\rho_{i}=\rho_{i 1}$ for all firms $i$. In another model we separately impose $\sigma_{i}^{2}=\sigma_{\mathrm{i} 4}^{2}$ for all firms $i$. Finally we consider a model with both of these restrictions. In this case the model reduces to a AR(1) model for quarterly earnings with quarterly dummies. Note that in these three restricted models, the dimension of the parameter space changes. In particular, the dimension of the vector $\bar{\theta}$ depends on the restrictions imposed on the autocorrelation parameters. 


\subsection{Testing hypotheses}

The four model variants presented above can be used to test for earnings management. For each of the models we compute the log marginal density [LMD], that is the density of the observed data. The model with the highest LMD fits best. LMDs can be formally used to test hypothesis the difference of the LMD of two models equals the $\log$ of the Bayes factor comparing the two models. A Bayes factor can be seen as a summary of the evidence for a hypothesis provided by the data (Kass and Raftery, 1995). We provide computational details in the appendix.

\section{Results}

We first compare the four different models. Table 1 shows the LMD for each of the model variants. The differences in LMD are very large. Translating these differences in terms of Bayes factors shows that there is overwhelming evidence that there are differences in autocorrelation and variances. In other words there is very strong evidence for earnings management. A difference in LMD larger than 5 is already considered to be decisive evidence (Kass and Raftery, 1995).

Insert Table 1 about here

Below we will only consider the model where the autocorrelation and the variances are allowed to differ across quarters. Figure 1 shows the posterior distribution of the population mean of the two autoregressive parameters as well as the posterior distribution of the difference. The figure clearly shows that the autoregressive coefficient for the first quarter is smaller than that for the other quarters.

Insert Tables 2 and 3 about here

The population parameters are also given in Tables 2 and 3. We can also look at the posterior results on the firm level. Figure 2 gives the histogram of the posterior means of the autocorrelation and variance parameters across firms. This figure also shows 
that in most cases $\rho_{1 \mathrm{i}}<\rho_{\mathrm{I}}$ and $\sigma_{i}^{2}<\sigma_{i 4}^{2}$. However the figure also shows that the opposite is also true for some firms. However, from the histogram of posterior means one cannot judge the significance of firm level parameters.

\section{Insert Table 4 about here}

In Table 4 we count the number of firms for which we find a significant difference in the parameters. For $29 \%$ of the firms we find that the correlation between the first quarter and the final quarter of the previous year is smaller than the correlation between any other combination of consecutive quarters. Next for $38 \%$ of the firms the conditional variance of the earnings in the fourth quarter is significantly larger than in the other quarters. 


\section{Conclusion}

The model can also simply be extended to see if things have changed over time, like Givoly and Hayn (2000) intend to test.

Also, one can include firm-specific characteristics in the second level, to see whether there is variation in earnings management across types of firms, like in Fok, Franses and van Dijk (Journal of Applied Econometrics). 


\section{Technical appendix}

For inference we write the model

$$
\begin{array}{ll}
Y_{i, 1, T}=\mu_{i, 1}+\rho_{1} Y_{i, 4, T-1}+\varepsilon_{i, 1, T} & \varepsilon_{i, 1, T} \sim N\left(0, \sigma_{i}^{2}\right) \\
Y_{i, 2, T}=\mu_{i, 2}+\rho Y_{i, 1, T}+\varepsilon_{i, 2, T} & \varepsilon_{i, 2, T} \sim N\left(0, \sigma_{i}^{2}\right) \\
Y_{i, 3, T}=\mu_{i, 3}+\rho Y_{i, 2, T}+\varepsilon_{i, 3, T} & \varepsilon_{i, 3, T} \sim N\left(0, \sigma_{i}^{2}\right) \\
Y_{i, 4, T}=\mu_{i, 4}+\rho Y_{i, 3, T}+\varepsilon_{i, 4, T} & \varepsilon_{i, 4, T} \sim N\left(0, \sigma_{i 4}^{2}\right)
\end{array}
$$

as

$$
\begin{aligned}
y_{i} & =\left(\begin{array}{l}
y_{i 2} \\
y_{i 3} \\
y_{i 4} \\
y_{i 5} \\
\vdots \\
y_{i n}
\end{array}\right)=\left(\begin{array}{ll}
D_{i}^{c} & D_{i}^{l} * y_{i,-1}
\end{array}\right) \theta_{i}+\left(\begin{array}{l}
\varepsilon_{i 2} \\
\varepsilon_{i 3} \\
\varepsilon_{i 4} \\
\varepsilon_{i 5} \\
\vdots \\
\varepsilon_{i n}
\end{array}\right) \\
& =X_{i} \theta_{i}+\varepsilon_{i}, \text { with } \varepsilon_{i} \sim N\left(0, \operatorname{diag}\left(D_{i}^{\varepsilon} \tau_{i}^{2}\right)\right),
\end{aligned}
$$

where $\theta_{i}$ collects all firm specific parameters, and where $\tau_{i}^{2}=\left(\sigma_{i}^{2}, \sigma_{4 i}{ }^{2}\right)^{\prime}$. The matrices $\mathrm{D}_{\mathrm{i}}^{\mathrm{c}}, \mathrm{D}_{\mathrm{i}}{ }^{1}$ and $\mathrm{D}_{\mathrm{i}}{ }^{\varepsilon}$ collect all necessary quarterly dummies, that is,

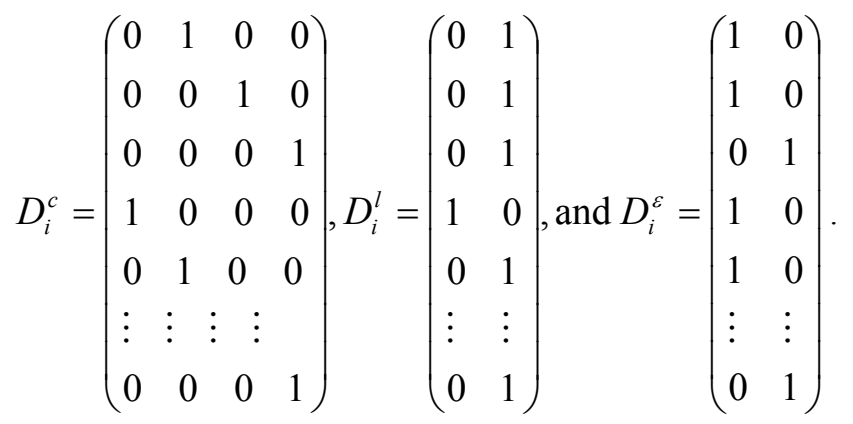

Note that we start with an observation in quarter 2, as we condition on the first observation. The vector $y_{i,-1}$ gives the lagged dependent variable. Finally the operator $*$ is defines as the element by element product, for example, for $D_{i}^{l} * y_{i,-}$ we multiply each element in the $i$-th row of $D_{i}^{l}$ with the $i$-th element of the vector $y_{i,-1}$.

We now summarize the model by

$$
\begin{aligned}
y_{i} & =X_{i} \theta_{i}+\varepsilon_{i} \\
\varepsilon_{i} & \sim N\left(0, \operatorname{diag}\left(D_{i}^{\varepsilon} \tau_{i}^{2}\right)\right) \\
\theta_{i} & \sim N(\bar{\theta}, \Sigma) .
\end{aligned}
$$

The model is completed with the following prior distributions 


$$
\begin{aligned}
& \Sigma \sim I W\left(V_{0}, v\right) \\
& \bar{\theta} \mid \Sigma \sim N(0, g \Sigma) \\
& \sigma_{i}^{2} \sim s^{2} / \chi^{2}(\varpi) \\
& \sigma_{4 i}^{2} \sim s^{2} / \chi^{2}(\varpi) .
\end{aligned}
$$

These distributions are of the natural conjugate form. We set the parameters of these distributions such that we have a proper but not very informative prior. Specifically, we set $\varpi$ and $s^{2}$ to 1 . The prior distribution for $\Sigma$ is an inverted Whishart with $\mathrm{V}_{0}$ equal to an identity matrix and degrees $v$ equal dimension of $\Sigma+3$. For the prior distribution of $\bar{\theta}$ conditional on $\Sigma$ we set $g$ equal to the number of firms $(\mathrm{K})$.

Given the above notation, the sampling steps for a Gibbs sampler are relatively straightforward. Below we specify the sampling scheme together with the corresponding distributions.

1. Draw $\theta_{i} \mid y_{i}, \bar{\theta}, \Sigma, \tau_{i}^{2}$ for all $i=1, \ldots K$

Based on (A1) we can sample

$$
\theta_{i} \mid y_{i}, \bar{\theta}, \Sigma, \tau_{i}^{2} \sim N\left(\left(X_{i}^{*} \cdot X_{i}^{*}+\Sigma^{-1}\right)^{-1}\left(X_{i}^{*} y_{i}^{*}+\Sigma^{-1} \bar{\theta}\right),\left(X_{i}^{*} \cdot X_{i}^{*}+\Sigma^{-1}\right)^{-1}\right),
$$

where

$$
y_{i}^{*}=\operatorname{diag}\left(D_{i}^{\varepsilon}\left(\frac{1}{\sigma_{i}}, \frac{1}{\sigma_{4 i}}\right)^{\prime}\right) y_{i} \text {, and } X_{i}^{*}=\operatorname{diag}\left(D_{i}^{\varepsilon}\left(\frac{1}{\sigma_{i}}, \frac{1}{\sigma_{4 i}}\right)^{\prime}\right) X_{i} .
$$

2. Draw $\sigma_{i}^{2}, \sigma_{4 i}^{2} \mid y_{i}, \theta_{i}$ for all $i=1, \ldots, K$

For each firm we sample the first variance as $\left(\sum_{t \mid S_{i t} \neq 4} e_{i t}^{2}+s^{2}\right) / \chi^{2}\left(\varpi+n_{i 1}+n_{i 2}+n_{i 3}\right)$, where the denominator denotes a draw from a $\chi^{2}$ distribution with the given number of degrees of freedom and $e_{i t}$ denotes the $t$-th element of $y_{i}-X_{i} \theta_{i}$. The second variance is sampled as $\left(\sum_{t \mid s_{i t}=4} e_{i t}^{2}+s^{2}\right) / \chi^{2}\left(\varpi+n_{i 4}\right)$.

\section{Draw $\Sigma, \bar{\theta} \mid \theta_{1}, \ldots, \theta_{K}$}

Using standard results for the multivariate regression model (see for example, Zellner 1971 or Rossi et $a l, 2005)$ we draw $\Sigma$ from an Inverted Wishart distribution with location $\left(\widetilde{E}^{\prime} \widetilde{E}+V_{0}\right)$ and degrees of freedom $(\mathrm{K}+v)$, where $\widetilde{E}=\left(\left(\theta_{1}-\widetilde{\theta}\right), \ldots\left(\theta_{K}-\widetilde{\theta}\right)\right)^{\prime}$ and $\widetilde{\theta}=\frac{1}{K+\frac{1}{g}}\left(\sum_{i=1}^{K} \theta_{i}\right)$. Conditional on $\Sigma$ we draw $\bar{\theta}$ from $N\left(\tilde{\theta}, \frac{1}{K+\frac{1}{g}} \Sigma\right)$

\section{TO DO: CALCULATION OF LMD}


Table 1: Comparison of model fit

\begin{tabular}{llc}
\hline Autocorrelation & Variance & Log Marginal Density \\
\hline Common & Common & -38771 \\
Common & Different & -35758 \\
Different & Common & -38361 \\
Different & Different & -35125 \\
\hline
\end{tabular}


Table 2: Posterior results of population parameters

\begin{tabular}{cccc}
\hline Parameter & Posterior mean & Posterior variance & \\
\hline $\bar{\rho}_{1}$ & 0.339 & $(0.022)$ & $* * *$ \\
$\bar{\rho}$ & 0.488 & $(0.018)$ & $* * *$ \\
$\alpha_{1}$ & 0.021 & $(0.017)$ & \\
$\alpha_{2}$ & 0.051 & $(0.016)$ & $* * *$ \\
$\alpha_{3}$ & 0.069 & $(0.019)$ & $* * *$ \\
$\alpha_{4}$ & -0.132 & $(0.019)$ & $* * *$ \\
\hline$* * *, * *, *: 0$ not contained in the $99,95,90 \%$ highest posterior density region, respectively
\end{tabular}


Table 3: Posterior mean of the covariance matrix

\begin{tabular}{lllllll}
\hline & $\bar{\rho}_{1}$ & $\bar{\rho}$ & $\alpha_{1}$ & $\alpha_{2}$ & $\alpha_{3}$ & $\alpha_{4}$ \\
\hline $\bar{\rho}_{1}$ & 0.143 & 0.058 & 0.008 & 0.002 & -0.025 & 0.031 \\
$\bar{\rho}$ & 0.058 & 0.110 & -0.010 & 0.020 & -0.016 & 0.015 \\
$\alpha_{1}$ & 0.008 & -0.010 & 0.088 & -0.048 & -0.034 & -0.003 \\
$\alpha_{2}$ & 0.002 & 0.020 & -0.048 & 0.070 & -0.007 & -0.005 \\
$\alpha_{3}$ & -0.025 & -0.016 & -0.034 & -0.007 & 0.114 & -0.061 \\
$\alpha_{4}$ & 0.031 & 0.015 & -0.003 & -0.005 & -0.061 & 0.090 \\
\hline
\end{tabular}


Table 4: Significance and sign of firm level differences of autocorrelation $\left(\rho_{1 i^{-}} \rho_{\mathrm{i}}\right)$ and firm level difference in variance $\left(\sigma_{i}^{2}<\sigma_{i 4}^{2}\right)$. Significant should be read as that 0 is not contained in the $95 \%$ highest posterior density region.

\begin{tabular}{lcccccc}
\hline \multirow{2}{*}{ Classification } & \multicolumn{2}{l}{ Autocorrelation } & & \multicolumn{2}{l}{ Variance } \\
\cline { 2 - 3 } \cline { 5 - 6 } & $\begin{array}{c}\text { Number } \\
\text { of firms }\end{array}$ & Percentage & & $\begin{array}{l}\text { Number } \\
\text { of firms }\end{array}$ & Percentage \\
\hline Significant and negative & 113 & 29 & & 147 & 38 \\
Not significant and negative & 163 & 42 & & 107 & 27 \\
Not significant and positive & 102 & 26 & & 76 & 20 \\
Significant and positive & 12 & 3 & & 60 & 15 \\
\hline
\end{tabular}


Table 5: Summary statistics across firms of posterior mean of autocorrelation and variance parameters

\begin{tabular}{|l|c|c|c|c|}
\hline & $\mathrm{E}\left[\rho_{\mathrm{i} 1} \mid \mathrm{Y}\right]$ & $\mathrm{E}\left[\rho_{\mathrm{i}} \mid \mathrm{Y}\right]$ & $\mathrm{E}\left[\sigma_{i}^{2} \mid \mathrm{Y}\right]$ & $\mathrm{E}\left[\sigma_{i 4}^{2} \mid \mathrm{Y}\right]$ \\
\hline Minimum & -1.102 & -0.218 & 0.025 & 0.057 \\
\hline Mean & 0.339 & 0.489 & 0.574 & 1.207 \\
\hline Maximum & 1.351 & 1.239 & 1.428 & 4.253 \\
\hline Variance & 0.113 & 0.095 & 0.130 & 1.014 \\
\hline
\end{tabular}



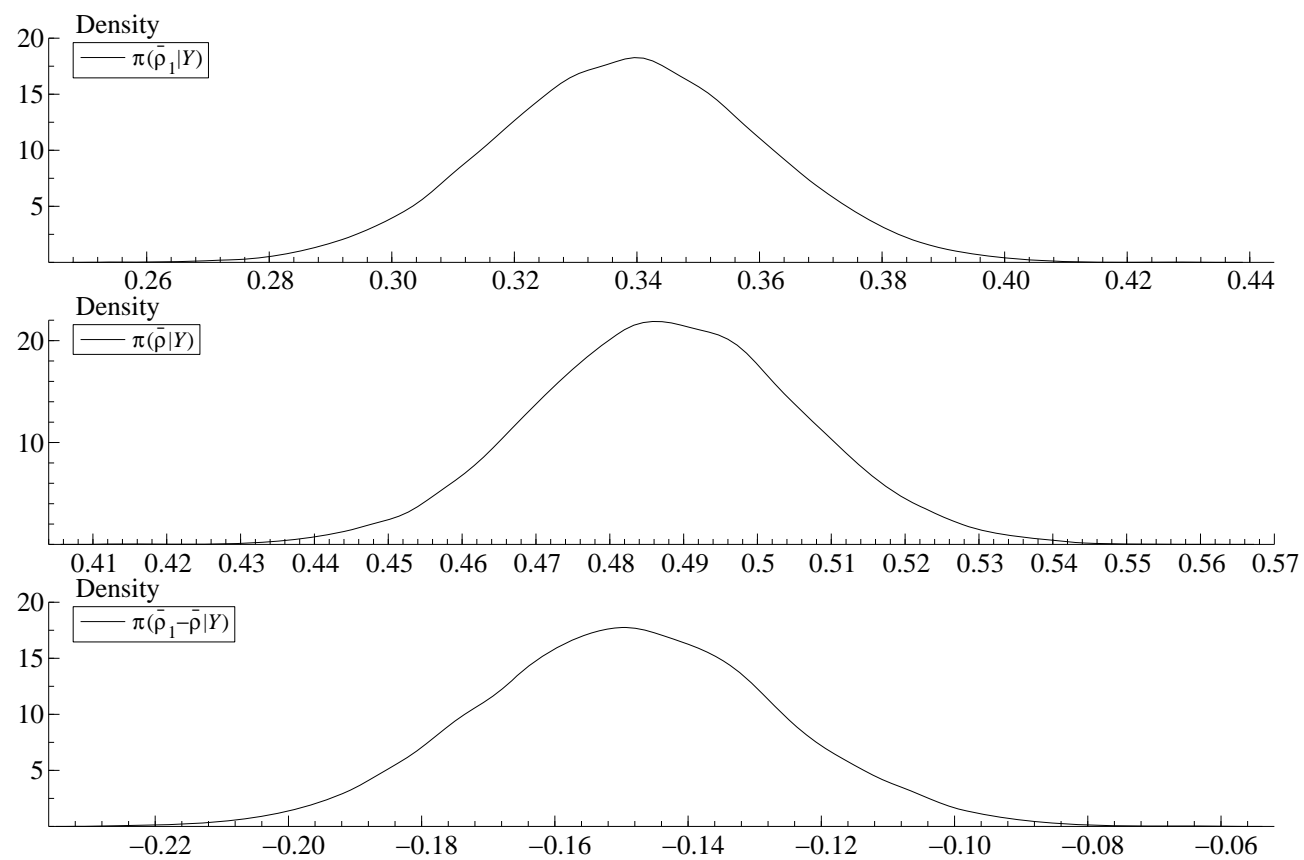

Figure 1: Posterior distribution of population mean autocorrelation parameters 

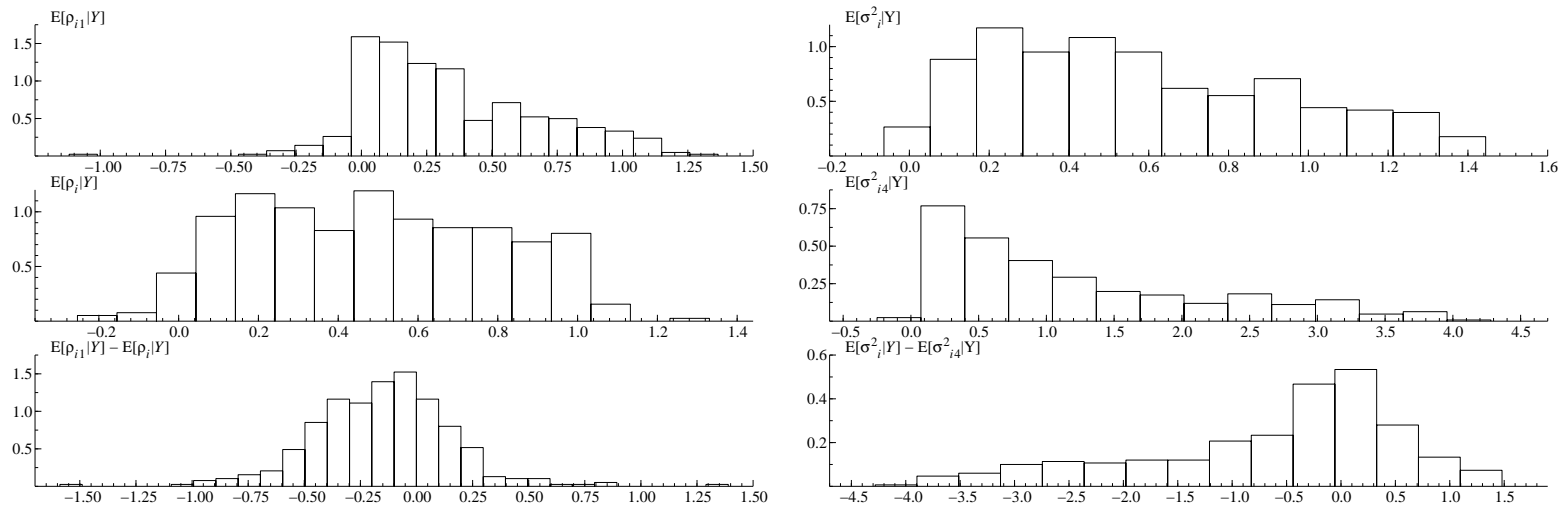

Figure 2: Histogram of posterior means across firms 

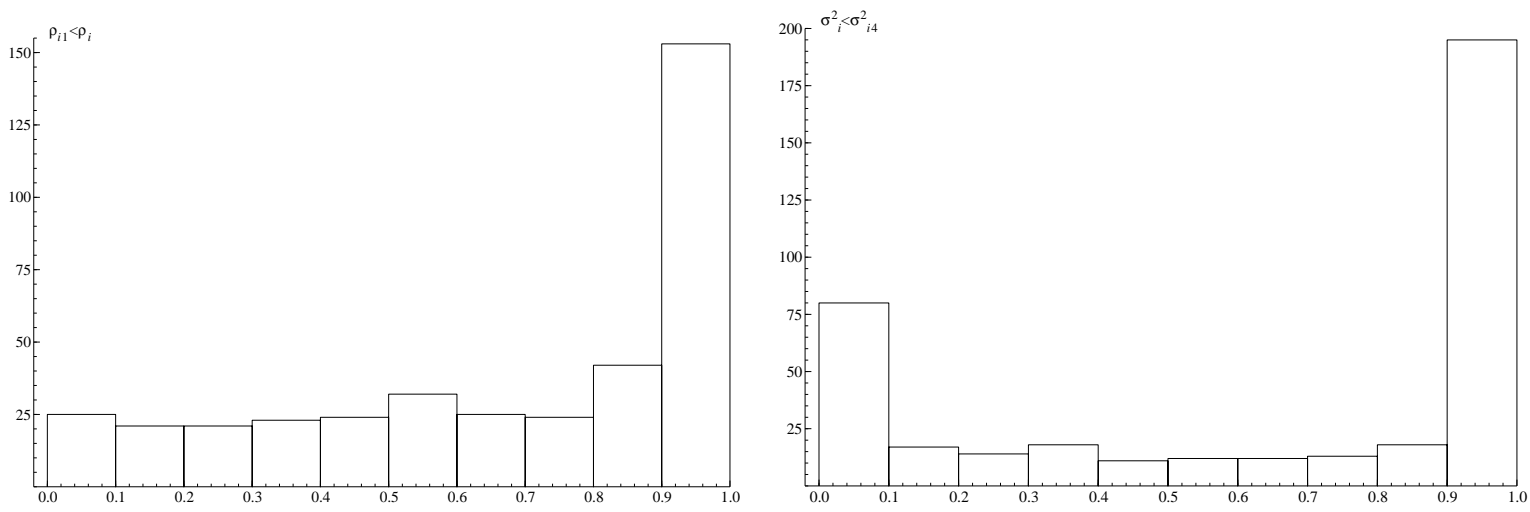

Figure 3: Histograms of the posterior probability that $\rho_{\mathrm{i} 1}<\rho_{\mathrm{i}}$ (left) and $\sigma_{i}^{2}<\sigma_{i 4}^{2}$ (right) across firms 


\section{References}

Burgstahler, D., Dichev, I.D., 1997, Earnings management to avoid earnings decreases and losses. Journal of Accounting and Economics 24, 99-126.

Collins, W.A., Hopwood, W.S. McKeown, J.C., 1984, The predictability of interim earnings over alternative quarters. Journal of Accounting Research 22, 467-479.

Das, S., Shroff, P.K., Zhang, H., 2007, Quarterly earnings patterns and earnings management. Working paper, University of Illinois at Chicago.

DeGeorge, F., Patel, J. Zeckhauser, R., 1999, Earnings management to exceed thresholds. Journal of Business 72, 1-33.

Franses, P.H, 1996, Periodicity and stochastic trends in economic time series. Oxford: Oxford University Press.

Franses, P.H., Paap, R. (2004), Periodic time series models, Oxford: Oxford University Press.

Geman, S., Geman D. (1984), Stochastic Relaxations, Gibbs Distributions, and the Bayesian Restoration of Images, IEEE Transaction on Pattern Analysis and Machine Intelligence, 6 (6), 721-41.

Givoly, D., Hayn C., 2000, The changing time-series properties of earnings, cash flows and accruals: Has financial reporting become more conservative? Journal of Accounting and Economics 29, 287-320.

Hayn, C., 1995, The information content of losses. Journal of Accounting and Economics 20, 125-153.

Hayn, C., Gans Narayanamoorthy, G., Watts, R.L., 2007, How and why fourth quarter earnings differ from interim quarter earnings? Working paper, Yale University. 
Jacob, J., Jorgensen, B.N., 2007, Earnings management and accounting income aggregation. Journal of Accounting and Economics 43, 369-390.

Kass, R.E., and A.E. Raftery, 1995, Bayes Factors, Journal of the American Statistical Association, 90(430), 773-795.

Rossi, P.E., Allenby, G.M., and R. McCulloch, 2005, Bayesian Statistics and Marketing, Wiley Series in Probability and Statistics, West Sussex, England

Zellner, A., 1971, An Introduction to Bayesian Inference in Econometrics, John Wiley $\&$ Sons, New York 